\title{
Forehead Pseudoaneurysm after Falling Down: A Case Report
}

\section{Jinn-Rung Kuo ${ }^{1,2 *}$ and Tzu-Hui Pao ${ }^{3}$}

${ }^{1}$ Department of Neurosurgery, Chi-Mei Medical Center, Yong Kang, Tainan, Taiwan

${ }^{2}$ Department of Biotechnology, Southern Taiwan University of Science and Technology, Tainan, Taiwan

${ }^{3}$ Department of General Medicine, Chi-Mei Medical Center, Yong Kang, Tainan, Taiwan

\begin{abstract}
Forehead pseudoaneurysm after falling down is a rare condition. We report a case of a patient with a 2-month history of a forehead growing mass after falling down and bumping her right forehead. The 3-dimensional computed tomography (CT) angiography showed a hyperdense lesion with internal enhancing focus and supplying by one small branch of right superficial temporal artery (STA). Surgical resection of the pseudoaneurysm with coagulation of the feeding artery was performed. However, there was profuse bleeding complication while drainage tube removal, an emergent explored surgical hemostasis was done. In this article, in addition to presenting the clinical course of an unusual case, we wanted to give emphasis to that ligation of the proximal and distal vessels are necessary while surgical resection of the pseudoaneurysm.
\end{abstract}

Keywords: Pseudoaneurysm; Superficial temporal artery; Threedimensional CT angiography; Surgery

\section{Case Report}

A 75-year-old woman presented with a 2-month history of a growing mass in her forehead. Two months previously, she had fallen and bumped her right forehead. There was a contusion area on the right side of the forehead, and finally resolved three weeks after the trauma. However, a small pulsatile nodule persevered and increased in size gradually. The physical examination revealed a $15 \times 15 \mathrm{~mm}$ sized pulsatile mass on the right frontal region. The pulsation decreased while pressuring on the proximal part of the STA. Ultrasonography showed $15 \times 5 \mathrm{~mm}$ of the lesion with intralesional vascularity. CT and 3-dimensional CT angiography showed a hyperdense lesion, measuring $12 \times 16 \mathrm{~mm}$ with internal marked enhancing focus and supplying by one small branch of right STA (Figure 1). Based on above findings, pseudoaneurysm of the STA was suspected. We described the tract of branches of STA for operative planning (Figure 2). The pseudoaneurysm was dissected carefully by sharp dissection, and clear margin was noted (Figure 3). The distal feeding artery was cut and coagulated by bipolar coagulation. The pseudoaneurysm was totally removed. However, there was profuse bleeding while Combination Waste Vent (CWV) drain removal three days after operation. We performed emergent explored surgical hemostasis of the CWV tract and STA branches. Hemovac drain was inserted through a new tract. The patient recovered uneventfully, and there was no bleeding or

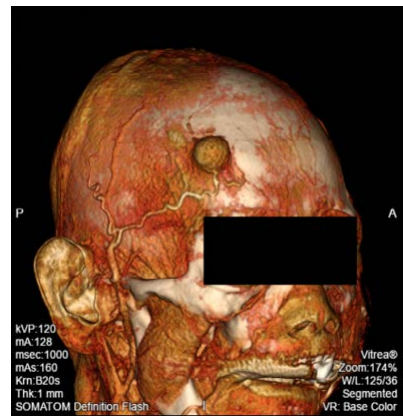

Figure 1: Three-dimensional CT angiography showed the $12 \times 16 \mathrm{~mm}$ pseudoaneurysm at right frontal scalp and supplying by one small branch of right frontal branch of STA. recurrence of the lesion. The final pathological findings confirmed a pseudoaneurysm (Figure 4).

\section{Discussion}

Pseudoaneurysm of the STA is an uncommon cause of a facial mass, in the majority of cases caused after head trauma [1]. Thomas Bartholin reported the first STA pseudoaneurysm case in 1740, and since then over 400 cases have been described [2]. It often caused after arterial trauma, resulting in arterial wall disruption, with development of a hematoma and pseudocapsule. Pressure of the blood flow on the wall bloated the cavity to form an aneurysm. Due to the formation of causes, the typical finding of pseudoaneurysm is a thrilling or pulsatile mass in the temporal region. Furthermore, the pulsation disappears while the proximal part of the STA is compressed [3].

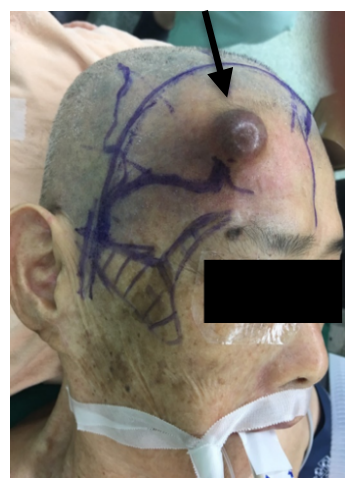

Figure 2: The preoperative view. The arrow shows the pseudoaneurysm in the right temporal area.

${ }^{*}$ Corresponding author: Jinn-Rung Kuo, MD, Department of Neurosurgery, ChiMei Medical Center, 901 Chung Hwa Road, Yong Kang, Tainan, Taiwan 710, Tel: 886-6-2812811; Fax: 886-6-2828928; E-mail: kuojinnrung@gmail.com

Received April 27, 2017; Accepted May 12, 2017; Published May 19, 2017

Citation: Kuo J, Pao TH (2017) Forehead Pseudoaneurysm after Falling Down: A Case Report. J Vasc Med Surg 5: 312. doi: 10.4172/2329-6925.1000312

Copyright: @ 2017 Kuo J, et al. This is an open-access article distributed under the terms of the Creative Commons Attribution License, which permits unrestricted use, distribution, and reproduction in any medium, provided the original author and source are credited. 


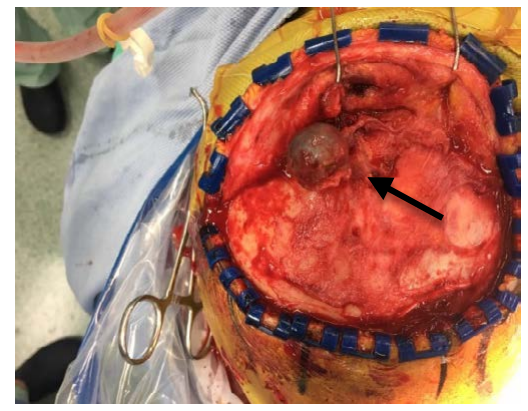

Figure 3: The intraoperative view. The arrowhead shows the pseudoaneurysm The arrow shows the supplying branch of right frontal branch of STA.

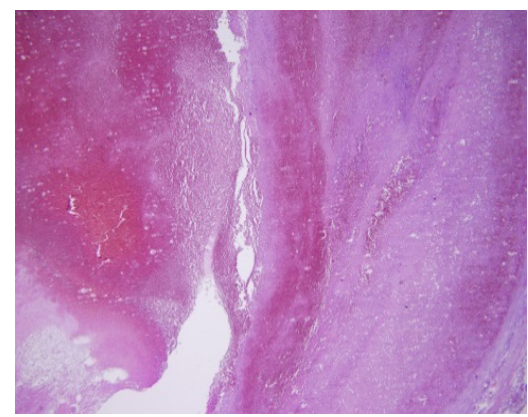

Figure 4: The section shows thrombus, consistent with pseudoaneurysm (40X).

The anterior superficial branch of the STA is most vulnerable because of its course. It paths superficially from the temporalis muscle to the lateral margin of the frontalis muscle. On the gap between the thin muscles, it lies directly against the skull with less defense from injury $[2,4,5]$. The case of this study is a pseudoaneurysm of anterior superficial branch of temporal artery, occurring within weeks after a blunt trauma due to falling down. The times window of a few hours to 10 years has been reported, although typically from weeks to months after injury [6], such as our case.

Pseudoaneurysm of STA can be diagnosed following a complete medical history and physical examination. However, it is recommended to confirm the diagnosis and to exclude other illnesses via image modalities including sonography, CT angiography and magnetic resonance imaging. Doppler ultrasound is a non-invasive method with high sensitivity and specificity. Selective arteriography is the most effective procedure for examination, but is invasive with risk of stroke $[2,4,7]$. CT and MR angiography are non-invasive technique, which can measure the true size of the STA pseudoaneurysm and the relationship of trunk and distal branches of STA. Moreover, 3-dimensional CT angiography has advantages for precisely depicting the morphology of the STA pseudoaneurysm, the adjacent soft tissue and osseous structures, which is helpful for planning of treatment strategy $[8,9]$.

STA aneurysms can be treated by surgical, embolization, percutaneous thrombin injection and conservative methods. Surgical resection and ligation is the most commonly described treatment of pseudoaneurysm of the STA, with high success rate and fewer complications $[10,11]$. The pseudoaneurysm was resected surgically with distal feeding artery cut and coagulated by bipolar coagulation in our case. Emergent complication with massive bleeding was occurred when the drainage tube was removed, which let us deeply understand the importance of ligation of the afferent and efferent vessels.

\section{Conclusion}

Superficial temporal artery pseudoaneurysm is an uncommon occurrence usually caused after traumatic event. Three-dimensional CT angiography precisely illustrated the morphology of pseudoaneurysm and relationship with the branch of STA and adjacent structures. The standard of the surgical treatment must include ligation of the afferent and efferent vessels in addition to resection of pseudoaneurysm.

\section{References}

1. Borah G, Han K (1996) Pseudoaneurysm of the anterior superficial temporal artery. Annals of Plastic Surgery 37: 650-653.

2. Menif E, Besbes G, Sahtout S, Zainine R, Hedhli M, et al. (2011) Post-traumatic pseudoaneurysm of the superficial temporal artery: A rare cause of facial mass. European Journal of Radiology Extra 79: e33-e35.

3. Chopra S, Gupta P, Jaiswal M, Vijay Sundar I, Sardana V (2012) Traumatic pseudoaneurysm of the superficial temporal artery: A case report and review of literature. The Indian Journal of Neurotrauma 9: 56-58.

4. Heran M, Mann G (2007) Percutaneous thrombin embolization of a posttraumatic superficial temporal artery pseudoaneurysm. Pediatric Radiology $37:$ 578-580.

5. Eoh W, Lee S, Jo K, Lee H (2010) Traumatic Pseudoaneurysm of the superficial temporal artery due to Gardner Traction. Journal of Korean Neurosurgical Society 48: 291

6. Stanley J, Hollier L, Shah D, Tilson M, Rutherford R, et al. (1991) Suggested standards for reporting on arterial aneurysms. Journal of Vascular Surgery 13 452-458.

7. Miyake H, Satoh K, Oka H, Tamura T, Masahira N, et al. (2010) Traumatic pseudoaneurysm of superficial temporal artery. The American Journal of Surgery 199: e1-e2.

8. Mannoji H, Kawashima M, Higashino T (2005) Three-Dimensional computed tomography Angiography for the investigation of superficial temporal artery Pseudoaneurysms. Neurologia medico-chirurgica 45: 152-155.

9. Kim S, Park S, Kim H, Park I (2008) Traumatic Pseudoaneurysm of the superficial temporal artery diagnosed by 3-dimensional CT Angiography. Journal of Korean Neurosurgical Society 43: 209.

10. Teijink J, Willigendael E, Klompenhouwer E, Thomassen I (2013) Treatment of temporal artery pseudoaneurysms. ISVS 22: 274-279.

11. Pajaj E, Alimehmeti R, Seferi A, Vyshka G (2016) Superficial temporal artery pseudoaneurysm presenting as a growing, pulsatile, and tender mass. Surgical Neurology International 7: 66 . 